\title{
X Congreso Ibérico de Agroingeniería

\section{Transferencia y acumulación de metales y metaloides tanto en lechuga como en plantas silvestres en suelos afectados por la minería metálica}

\author{
Martínez-Carlos J. ${ }^{1}$, Martínez-Martínez S. ${ }^{1}$, Zornoza R., Faz A. ${ }^{1}$, Gabarrón M, Soriano-Disla \\ M. ${ }^{1}$, Gómez-López M.D. ${ }^{1}$, Acosta J.A. ${ }^{1}$ \\ 1 Grupo de Investigación Gestión, Aprovechamiento y Recuperación de Suelos y Aguas; Departamento \\ de Ingeniería Agronómica; Universidad Politécnica de Cartagena, Paseo Alfonso XIII 48, 30203 \\ Cartagena, España; silvia.martinez@upct.es.
}

Resumen: La transferencia y acumulación de elementos tóxicos desde suelos contaminados hacia los cultivos o plantas silvestres es de especial interés tanto desde el punto de vista medioambiental como para la salud pública. En este estudio se evaluó la transferencia y acumulación de metales y metaloides tanto en plantas silvestres (Chamaerops humilis, Lavanda multífida, Olea europea sylvestris, Pistacia lentiscus, Rhamnus Lycioides) como en diferentes variedades de lechuga ("Romana", "Batavia" y "Lollo rosso"). El estudio se realizó en una zona afectada por la minería metálica del sureste de España. Las 3 variedades de lechuga fueron cultivadas en maceta bajo invernadero, utilizando tres tipos distintos de suelos agrícolas afectados por dicha minería. Para alcanzar los objetivos planteados se analizaron las concentraciones de $\mathrm{Cd}, \mathrm{Zn}, \mathrm{Pb}$ y As en suelo y planta (raíz, tallo, hoja y fruto), lo que permitió conocer tanto la acumulación de metal/oide en los diferentes órganos de las plantas, así como calcular los factores de transferencia suelo/planta y raíz/(tallo-hoja-fruto). Los resultados mostraron que el mayor riesgo de transferencia se observaba en las hojas de Lavandula multifida, mientras que la mayor acumulación se presentaba en las partes aéreas de Olea europea. En general, el elemento más móvil en el sistema suelo/planta fue el $\mathrm{Zn}$ seguido de $\mathrm{Pb}>$ $\mathrm{Cd}>\mathrm{As}$, siendo $\mathrm{Zn}$ seguido de $\mathrm{Pb}$ los más móviles dentro de la planta, principalmente hacia las hojas. Por el contrario, Pistacia lentiscus acumuló la mayor parte de los metales absorbidos en raíz, no transferirlos a la parte aérea, por lo que podría ser usadas como especie fitoestabilizadora. Por su parte, los resultados obtenidos en las variedades de lechuga mostraban que el menor riesgo de transferencia de metales se presentaba en la variedad de lechuga "Romana", mientras que Lollo rosso registraba una mayor transferencia de As y $\mathrm{Pb}, \mathrm{y}$ Batavia la variedad con una mayor transferencia de $\mathrm{Cd}$ y $\mathrm{Zn}$. Los contenidos de metales acumulados en las tres variedades estudiadas estaban por encima de los límites tolerables.

Palabras clave: riesgo ambiental, transferencia de metal, fitoestabilización, residuo minero, cultivo en invernadero.

\section{Introducción}

La contaminación del suelo es uno de los principales problemas medioambientales a escala global. La Sierra Minera, ubicada entre las ciudades de Cartagena y La Unión en el sureste de España, fue una de las zonas mineras más grandes del mundo, en ella se producía $\mathrm{Pb} / \mathrm{Zn}$ hasta finales de los años 90 del siglo pasado [1,2]. Con el desarrollo de la actividad minera, se 


\section{CONGRESO IBÉRICO DE AGROINGENIERÍA \\ X CONGRESSO IBÉRICO DE AGROENGENHARIA \\ 3 - 6 septiembre 2019, Huesca - España}

depositaron millones de toneladas de residuos mineros en más de 80 depósitos mineros. Estos depósitos se abandonaron después del cese de la actividad en 1991. La acumulación de residuos mineros es de gran preocupación debido al riesgo asociado al alto contenido de metales/oides tóxicos. Además, estos depósitos se caracterizar por presentar baja fertilidad, bajo contenido de materia orgánica y alta acidez. Por lo tanto, el establecimiento de vegetación nativa es muy difícil a menos que se apliquen enmiendas orgánicas y/o inorgánicas $[3,4,5,6]$. Y esta ausencia de especies vegetales en los depósitos mineros favorece la dispersión potencial de metal/oides a los suelos naturales y agrícolas próximos, que pueden ser acumulados por las plantas que crecen en estos suelos y ser transferidos a la cadena trófica desencadenando posibles riesgos ambientales y para la salud humana. Para evitar esta dispersión de metales/oides, se podrían llevar a cabo técnicas de fitorremediación en los depósitos mineros abandonados. La fitorremediación se considera un método económico y ambientalmente sostenible para remediar los suelos contaminados [7]. Entre las técnicas de fitorremediación, la fitoestabilización asistida puede ser una solución para reducir el riesgo de dispersión de contaminantes [8,9]. La fitoestabilización asistida consiste en el uso de plantas junto a la incorporación de enmiendas orgánicas / inorgánicas. Las plantas elegidas deben desarrollar un extenso sistema radicular y una gran cantidad de biomasa en presencia de altas concentraciones de metal/oide. Al mismo tiempo, la translocación de los metales desde las raíces debe ser lo más lenta posible, limitando la transferencia de metales a la cadena trófica [10]. Después de la fitorremediación, el establecimiento de una cubierta vegetal natural o inducida contribuye a mejorar la calidad física. Esto favorece el desarrollo de los suelos porque la rizosfera es una fuente de nutrientes y un refugio para los microorganismos $[11,12]$.

Se han propuesto varias enmiendas para estabilizar el metal(loide) en los suelos [13]. Las enmiendas orgánicas y los materiales ricos en carbonatos se han utilizado para reducir la biodisponibilidad de los metales y restaurar la función ecológica de los suelos contaminados $[14,15]$. La reducción de la biodisponibilidad del metal por los materiales orgánicos se debe a la adsorción sobre superficies sólidas y la complejación con sustancias húmicas. Este proceso de adsorción depende del metal particular, el tipo de suelo, el grado de materia orgánica, la humificación, el contenido de metales y sales, los efectos de la materia orgánica en el potencial redox y el pH [11]. El uso de subproductos es una forma de reducir la eliminación de desechos y revalorizarlos. También contribuyen a compensar la escasez de materia orgánica y aumentan la fertilidad, impiden la degradación del suelo, contribuyen al secuestro de carbono, mejoran la calidad del suelo y aumentan la biodiversidad.

La transferencia de metales/oides a cultivos cercanos a los depósitos mineros supone un riesgo para la salud pública. La acción erosiva incorpora metales a las tierras de cultivo que son tomados por la planta y que puede afectar de distinta manera según la tasa de consumo, la especie vegetal, las propiedades y composición química del suelo [16]. Las lechugas son un ingrediente muy común en la dieta mediterránea y acumulan más metales en sus partes comestibles que otras especies como los tomates o las habas. Esto es debido a que las plantas presentan mayor concentración en sus raíces, tallos y hojas que en sus frutos [17]. Estudios recientes en la Sierra Minera han encontrado suelos de cultivo de lechugas con altas concentraciones de $\mathrm{Pb}$ y $\mathrm{Zn}$ [18].

El objetivo del estudio fue (i) evaluar la transferencia de $\mathrm{Cd}, \mathrm{Zn}, \mathrm{Pb}$ y As del suelo a las plantas en un área natural de la Rambla del Avenque, (ii) determinar la transferencia de $\mathrm{Cd}, \mathrm{Zn}$, $\mathrm{Pb}$ y $\mathrm{As}$ en plantas hortícolas cultivadas en maceta con suelo tomado de áreas agrícolas próximas a los depósitos mineros de la Rambla del Avenque y bajo invernadero; (iii) seleccionar las mejores especies de plantas de la zona natural para la fitoestabilización asistida de depósitos mineros. 


\section{CONGRESO IBÉRICO DE AGROINGENIERÍA \\ X CONGRESSO IBÉRICO DE AGROENGENHARIA \\ 3 - 6 septiembre 2019, Huesca - España}

\section{Materiales y métodos}

\section{1. Área de estudio y diseño experimental}

Se cogieron muestras de suelo y planta silvestre en un área forestal de la Rambla del Avenque cercana a cinco depósitos de estériles mineros de la Sierra Minera de Cartagena-La Unión (Figura 1). Estos depósitos presentan un pH ácido y alta salinidad [8], que unido a las aridez característica del clima mediterráneo: escasez de lluvias ( $275 \mathrm{~mm}$ anual), temperatura templada $\left(18^{\circ} \mathrm{C}\right)$ y alta evapotranspiración $(>900 \mathrm{~mm}$ anual $)$, dificultan el establecimiento de la vegetación. La acción erosiva del viento traslada las partículas de los residuos mineros a las zonas agrícolas y forestales próximas y éstos son incorporados por el suelo y la planta.

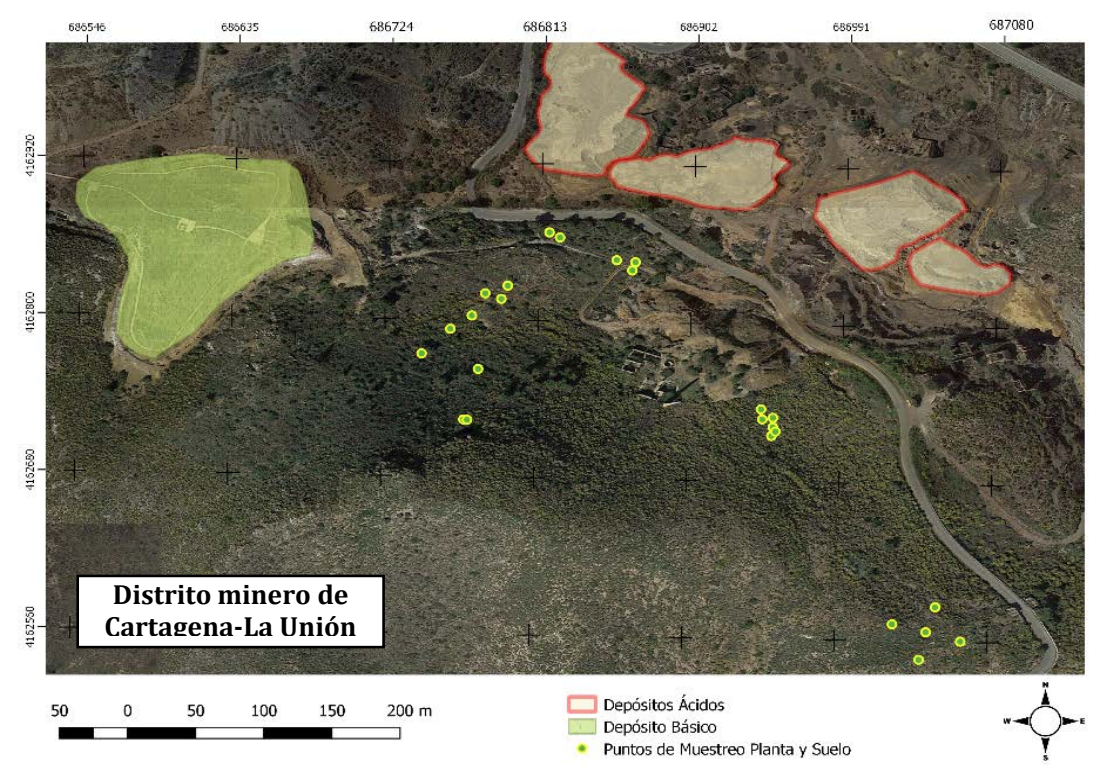

Figura 1. Mapa de localización de las muestras de suelo y planta en la zona forestal de la Rambla del Avenque.

En el área forestal localizada en la Rambla del Avenque se seleccionaron cinco especies vegetales representativas por criterio de abundancia (Chamaerops humilis- $\mathrm{CH}$, Lavandula multifida-LM, Olea europaea var. Sylvestris-OE, Pistacia lentiscus-PL, Rhamnus lycioides-RL), y se tomaron un total de 25 muestras de plantas ( 5 réplicas por especie) con sus respectivos suelos rizosféricos y no rizosféricos.

Además, se seleccionaron tres suelos agrícolas representativos de las zonas de cultivo próximas a la zona minera de estudio para cultivar 3 variedades de lechuga (Romana, Lollo rosso y Batavia) en macetas bajo invernadero (Foto 1). Se realizaron cinco réplicas por variedad y por tipo de suelo.

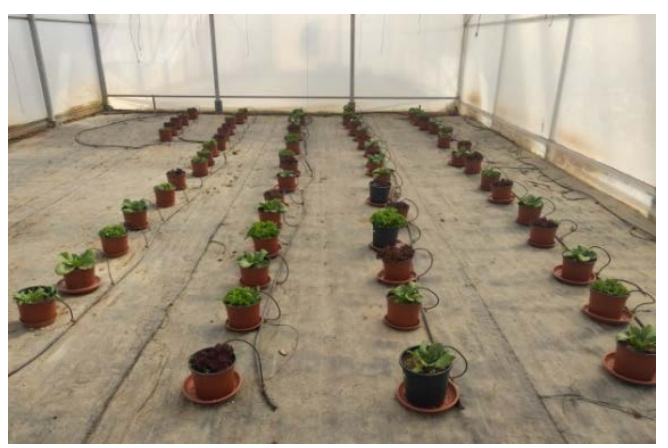

Foto 1. Cultivo de lechugas en el interior del invernadero. 


\section{CONGRESO IBÉRICO DE AGROINGENIERÍA \\ X CONGRESSO IBÉRICO DE AGROENGENHARIA \\ 3 - 6 septiembre 2019, Huesca - España}

\subsection{Métodos analíticos}

El suelo rizosférico se obtuvo removiendo el material adherido a las raíces de los ejemplares de planta recolectados. Todas las muestras fueron secadas a $45^{\circ} \mathrm{C}$ durante 7 días, pasadas por un tamiz de luz $2 \mathrm{~mm}$ y molido en mortero tipo Ágata (RetschRM 100).

Las plantas procedentes de los suelos naturales y las lechugas cultivadas bajo invernadero fueron llevadas al laboratorio donde se separaron en raíz, tallo, frutos y hojas, a continuación se lavaron con agua desionizada y se secaron al horno hasta peso constante a $45^{\circ} \mathrm{C}$ durante 5 días.

Para las muestras de suelo se prepararon soluciones con agua destilada/suelo [19] para la medida de $\mathrm{pH}$ (ratio 1:1) y conductividad eléctrica (1:5). La concentración total de metal en las muestras de planta y suelo se obtuvo por digestión con microondas, método US-EPA 3052[20] , utilizando $0,5 \mathrm{~g}$ de planta en $\mathrm{HNO} / \mathrm{H} 2 \mathrm{O} 2 / \mathrm{HCl}$ y en $\mathrm{HNO} 3$ en el caso de las muestras de suelo. Por su parte, la fracción biodisponible de metales se obtuvo de las fases lábiles del procedimiento de extracción secuencial de Tessier et al. [21] modificado por Li et al. [22]. Para la extracción secuencial del As se siguió el método propuesto por Shiowatana et al. [23]. Todas las concentraciones de metales/oides totales y biodisponibles se midieron mediante ICP-MS (Agilent 7500CE).

Se llevaron a cabo cálculos de los factores bioacumulación (BCF) [24] y el de translocación (TF) [25], [26].

$$
\text { Factor de bioacumulación }(\mathrm{BCF})=\frac{\text { Concentración de metal en cada parte de la planta }}{\text { Concentración de metal biodisponible en el suelo }}
$$

Factor de translocación $(\mathrm{TF})=\frac{\text { Concentración de metal en la parte aérea (tallos, frutos y hojas). }}{\text { Concentración de metal en raíces }}$

El factor de bioacumulación (BCF) se utiliza para medir la capacidad de captación de un metal por una planta (raíz y parte aérea) con relación a su concentración en el suelo. Para las plantas, el BCF se utiliza como una medida de la eficiencia de acumulación de metales en biomasa. Aquellas especies exclusoras poseen BCF menores que 1, tanto menores cuanto mayor es su capacidad de exclusión. Por su parte, TFs mayores a 1 sugieren gran capacidad para transportar metales desde las raíces a los vástagos, explicada en su mayor parte por eficientes sistemas de transporte de metales [27] y, probablemente, por secuestro de metales en las vacuolas de las hojas y en el apoplasto [28].

\section{Resultados y discusión}

\subsection{Suelo forestal}

Los suelos de la zona forestal son ligeramente-moderadamente alcalinos $(7,7-8,1)$ e hipersalinos $\left(247,7-1460,6 \mathrm{dSm}^{-1}\right)$. Las concentraciones totales medias de metal/oide en estos suelos es de 7,91 mg kg-1 para Cd; 288,7 mg kg-1 para As; $2882,2 \mathrm{mg} \mathrm{kg}^{-1}$ para Pb y $1865,4 \mathrm{mg} \mathrm{kg}^{-1}$ en el caso del $\mathrm{Zn}$, todas ellas superiores a los valores de fondo de metal/oide establecidos por Martínez y Pérez [29] en esta zona de estudio. Por su parte, la movilidad de los metales disminuye conforme $\mathrm{Cd}>\mathrm{As}>\mathrm{Pb}>\mathrm{Zn}$.

Respecto a los resultados de planta, los metales que más se acumularon fueron $\mathrm{Pb}$ y $\mathrm{Zn}$, siendo las raíces de PL las que presentaban mayores contenidos de $\mathrm{Pb}$ y las hojas de LM las mayores concentraciones de ambos metales. Valores de $\mathrm{BCF}>1$ se registraron en las raíces de PL para $\mathrm{Pb}$ y en las hojas de LM para $\mathrm{Pb}$ y $\mathrm{Zn}$, lo que indica que estas dos especies son potencialmente hiperacumuladoras [29]. La acumulación en las partes comestibles $(\mathrm{BCF}>1)$ puede suponer un riesgo de incorporación a la cadena trófica a la vez que hace a las especies una buena elección para aplicar técnicas fitoextractivas [30] por haber desarrollado resistencia a la presencia de metales en sus tejidos 


\section{CONGRESO IBÉRICO DE AGROINGENIERÍA \\ X CONGRESSO IBÉRICO DE AGROENGENHARIA \\ 3 - 6 septiembre 2019, Huesca - España}

y ser plantas acumuladoras. Sin embargo, si la planta presenta un $B C F>1$ en la parte radicular podría ser una buena candidata para ser utilizada en la técnica de fitoestabilización, siempre y cuando no se produzca una translocación del metal/oide a la parte aérea.

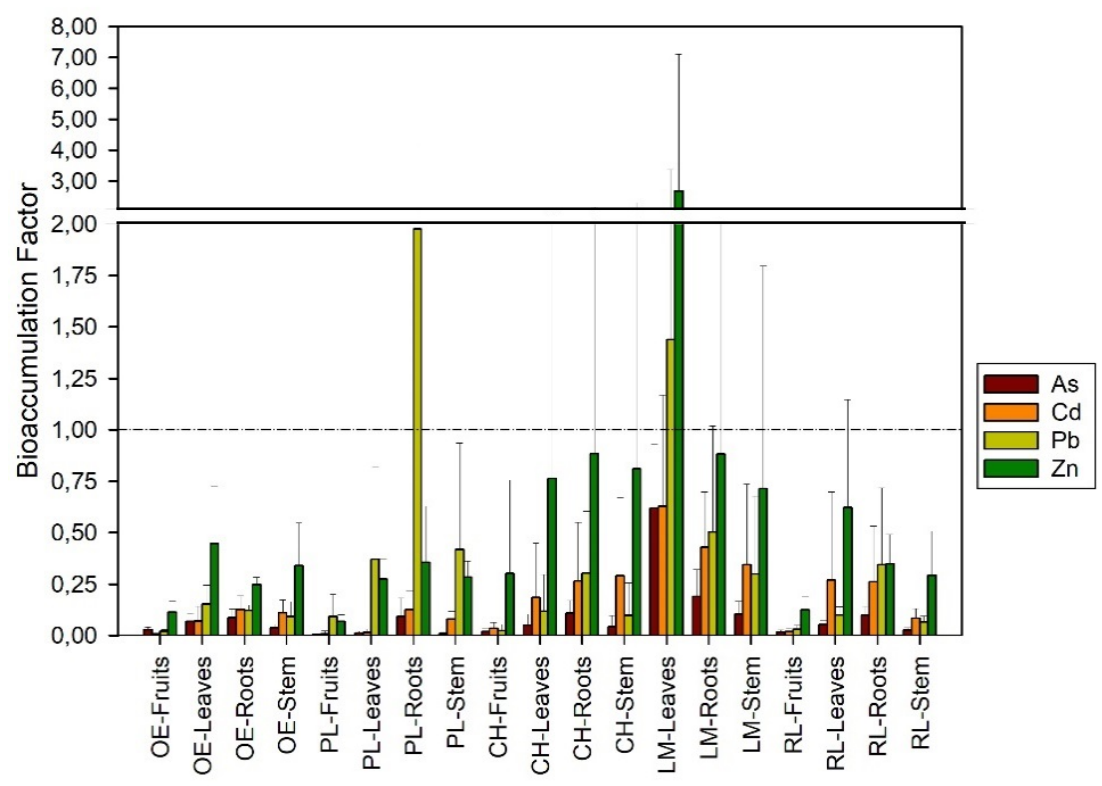

Figura 2a. Factor de bioacumulación (BCF) en plantas procedentes de una zona natural próxima a la Rambla del Avenque.

Las hojas de LM, además, presentaron altos valores del factor de translocación (TF>1) para $\mathrm{Pb}, \mathrm{Zn}$ y $\mathrm{Cd}$, y sobre todo para As que se aproxima a un valor de 4 , mientras que las hojas de RL para Cd y Zn. OE y PL también translocan Zn a sus hojas y tallos (Figura $2 b$ ).

PL sería la especie óptima para fitoestabilización por acumular Pb en sus raíces pero no transferirlo a la parte comestible, contribuyendo a la inmovilización de los metales en el suelo [31][32].

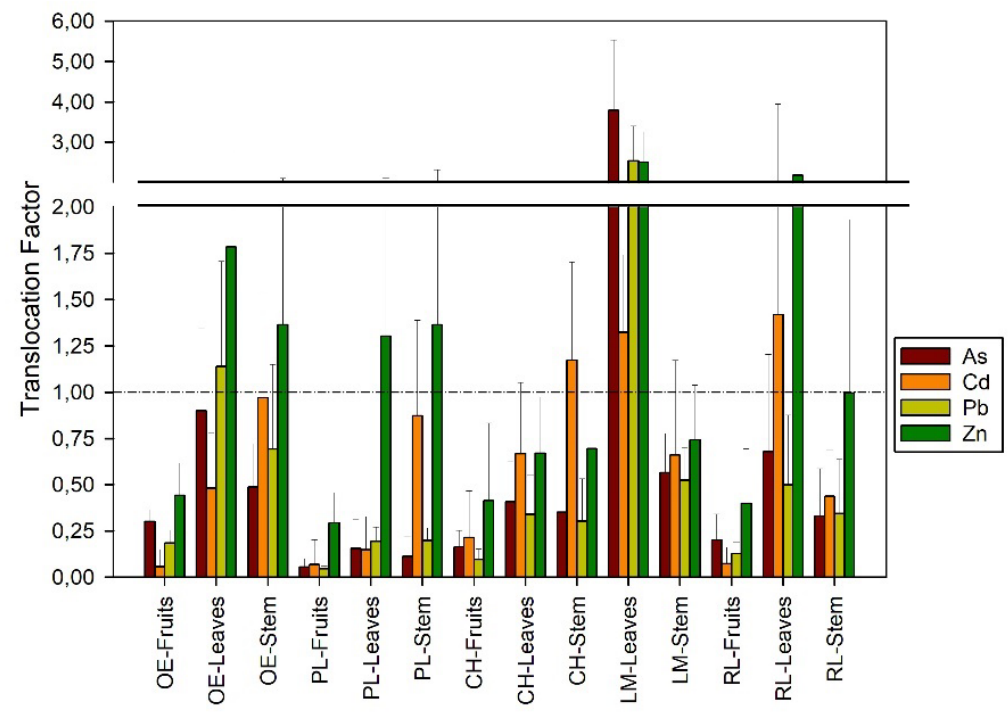

Figura 2b. Factor de translocación (TF) en plantas procedentes de una zona natural próxima a la Rambla del Avenque. 


\section{CONGRESO IBÉRICO DE AGROINGENIERÍA \\ X CONGRESSO IBÉRICO DE AGROENGENHARIA \\ 3 - 6 septiembre 2019, Huesca - España}

\subsection{Suelo agrícola}

Los suelos agrícolas en los que se cultivaron las lechugas presentaron un valor de $\mathrm{pH}$ neutro $(7,5)$ y conductividad eléctrica diferente según las zona de muestreo, siendo suelos ligeramente salinos los de la zona $1\left(2,6 \mathrm{dS} \mathrm{m}^{-1}\right)$ y no salinos los de las zonas $2-3\left(<1 \mathrm{dS} \mathrm{m}^{-1}\right)$. Con respecto al contenido en metales, el de mayor presencia fue $\mathrm{Zn}\left(1592-3112 \mathrm{mg} \mathrm{kg}^{-1}\right)$ seguido de $\mathrm{Pb}$ (596-1035 $\left.\mathrm{mg} \mathrm{kg}^{-1}\right)$, As (19-52 $\left.\mathrm{mg} \mathrm{kg}^{-1}\right)$ y Cd (2-8 $\left.\mathrm{mg} \mathrm{kg}^{-1}\right)$, siendo los suelos de la zona 1 los que presentaron mayores concentraciones seguidos de los de la zona $3 \mathrm{y}$, en último lugar, los de la zona 2.

Las lechugas cultivadas en el suelo de la Zona 1 presentaron las concentraciones más altas de metales (Tabla 1) superando los valores obtenidos por otros autores en lechugas cultivadas en zonas mineras [17, 18, 27]. Del mismo modo todos los datos obtenidos superaron los límites tolerables propuestos por Kabata-Pendias y Pendias [34]. En la Zona 2, esos límites fueron superados excepto para Cd (en Lollo Rosso) y Zn (en Lollo Rosso y Romana). Por su parte, en las lechugas cultivadas con suelo agrícola de la zona 3 el metal que más se acumulaba era Zn, seguido de $\mathrm{Pb}$ y por último $\mathrm{Cd}$ y As. Aunque las concentraciones de $\mathrm{Zn}$ fueron considerables no sobrepasaban los valores tolerables para su consumo. En este estudio, el mecanismo de entrada del metal hacia la planta fue vía radicular descartándose la vía foliar por las condiciones particulares de un cultivo en invernadero, y teniendo en cuenta que no fueron utilizados ningún herbicida, pesticida, el único imput fue el riego y la fertirrigación de N-P-K que se utliza usualmente para el cultivo de lechuga en el campo de Cartagena.

Tabla 1. 1a-Concentraciones de metales/oides en hojas de lechuga obtenidas en este estudio (mg $\mathrm{kg}^{-1}$ ); $1 \mathrm{~b}$ - Concentraciones de metales de referencia en cultivos agrícolas y 1c-Concentrationes de metales en lechugas cultivadas en zonas mineras o próximas a ellas.

\begin{tabular}{|c|c|c|c|c|c|}
\hline Variedad & Zona & $\begin{array}{c}\text { As } \\
\left(\mathrm{mgkg}^{-1}\right)\end{array}$ & $\begin{array}{c}\mathrm{Cd} \\
\left(\mathrm{mgkg}^{-1}\right)\end{array}$ & $\begin{array}{c}\mathrm{Pb} \\
\left(\mathrm{mgkg}^{-1}\right)\end{array}$ & $\begin{array}{c}\mathrm{Zn} \\
\left(\mathrm{mgkg}^{-1}\right)\end{array}$ \\
\hline & 1 & 1,50 & 3,37 & 22,49 & 141,3 \\
\hline \multirow[t]{3}{*}{${ }^{a}$ Batavia } & 2 & 0,59 & 0,67 & 5,54 & 51,61 \\
\hline & 3 & 0,55 & 1,52 & 6,17 & 73,33 \\
\hline & 1 & 4,46 & 1,82 & 67,66 & 215,8 \\
\hline \multirow[t]{3}{*}{${ }^{a}$ Lollo Rosso } & 2 & 1,00 & 0,43 & 10,21 & 33,37 \\
\hline & 3 & 1,32 & 0,85 & 24,33 & 62,95 \\
\hline & 1 & 1,21 & 2,36 & 14,50 & 70,52 \\
\hline \multirow[t]{2}{*}{ aRomana } & 2 & 0,31 & 0,31 & 1,67 & 20,49 \\
\hline & 3 & 0,22 & 0,78 & 2,05 & 33,61 \\
\hline${ }^{\mathrm{b}}$ Tolerable in agronomic crops [34]. & & 0,20 & $0,05-0,5$ & $0,5-10$ & $50-100$ \\
\hline 'Cobb et al. [17]. & & 5,47 & 1,61 & 29,80 & 60,00 \\
\hline 'Conesa et al. [18]. & & -- & -- & 10,00 & 90,00 \\
\hline 'Wang et al. [33] . & & -- & 1,00 & 15,00 & 300,00 \\
\hline
\end{tabular}

\section{Conclusiones}

La mayor acumulación de metales en la parte aérea de la planta la presentó Lavandula multifida, en concreto $\mathrm{Pb}$ y $\mathrm{Zn}$. Esto hace a la especie una buena elección para fitoextracción a la vez que supone un riesgo de incorporación a la cadena trófica. La mejor especie para llevar a 


\section{CONGRESO IBÉRICO DE AGROINGENIERÍA \\ X CONGRESSO IBÉRICO DE AGROENGENHARIA \\ 3 - 6 septiembre 2019, Huesca - España}

cabo trabajos de fitoestabilización fue Pistacia lentiscus, al acumular gran cantidad de metales en sus raíces y no transferirlo a sus tejidos comestibles.

Los metales más transferidos desde los suelos a la planta en la zona forestal fueron $\mathrm{Pb}$ y $\mathrm{Zn}$. Cd y As, aunque se encuentran también por encima de los niveles de fondo establecidos para la zona, no son incorporados por las plantas a sus tejidos y permanecen en su rizosfera. Se hace necesario profundizar en los estudios del efecto que tiene la rizosfera en la dinámica de los metales del suelo.

Las lechugas cultivadas con suelo agrícola próximo a la Sierra Minera Cartagena-La Unión estaban enriquecidas con metales, especialmente $\mathrm{Pb}$ y $\mathrm{Zn}$, comparado con lechugas de otras zonas mineras, superando todas ellas los límites tolerables para cultivos. La vía de entrada en las lechugas cultivadas en este estudio fue principalmente radicular, por las condiciones particulares de cultivo en invernadero, posiblemente, estas lechugas cultivadas al aire libre en las mismas zonas agrícolas donde se recolectó el suelo presentarían mayor concentración de metal/oide por la influencia del desarrollo de la actividad agrícola y por la deposición de metal/oide en los tejidos vegetales transportado por el viento desde zonas mineras próximas y urbanas.

\section{Agradecimientos}

Este trabajo ha sido cofinanciado por la Unión Europea a través de un proyecto Interreg SUDOE (SOILTAKECARE).

\section{Referencias}

1. Kabas S. et al. Effect of marble waste and pig slurry on the growth of native vegetation and heavy metal mobility in a mine tailing pond. Journal of Geochemical Exploration. 2012, volumen 123, (6976).

2. Faz A., Arnaldos R., Conesa H., García G. Soils affected by mining and industrial activities in Cartagena (SE Spain): classification problems. European Soil Bureau. 2001, volumen Research report 7, (165-170).

3. Madejón P., Pérez-de-Mora A., Burgos P., Cabrera F., Lepp N. W., Madejón E. Do amended, polluted soils require re-treatment for sustainable risk reduction? - Evidence from field experiments. Geoderma. 2010, volumen 159, (174-181).

4. Madejón E., de Mora A. P., Felipe E., Burgos P., Cabrera F. Soil amendments reduce trace element solubility in a contaminated soil and allow regrowth of natural vegetation. Environmental Pollution. 2006, volumen 139, (40-52).

5. García C., Lobo M. Rehabilitación de suelos degradados y contaminados mediante la aplicación de compost. Moreno Casco, J., Moral Herrero, R. (Eds.) Compostaje. Ediciones Mundi-Prensa, Madrid, España. 2007, , (425-448).

6. Pérez-de-Mora A., Burgos P., Madejón E., Cabrera F., Jaeckel P., Schloter M. Microbial community structure and function in a soil contaminated by heavy metals: effects of plant growth and different amendments. Soil Biology and Biochemistry. 2006, volumen 38, (327-341).

7. Wan X., Lei M., Chen T. Cost-benefit calculation of phytoremediation technology for heavy-metalcontaminated soil. Science of The Total Environment. 2016, volumen 563-564, (796-802).

8. Martínez-Martínez S., Acosta J. A., Cano A. F., Carmona D. M., Zornoza R., Cerda C. Assessment of the lead and zinc contents in natural soils and tailing ponds from the Cartagena-La Unión mining district, SE Spain. Journal of Geochemical Exploration. 2013, volumen 124, (166-175).

9. Zhao L., Li T., Yu H., Zhang X., Zheng Z. Effects of [S,S]-ethylenediaminedisuccinic acid and nitrilotriacetic acid on the efficiency of $\mathrm{Pb}$ phytostabilization by Athyrium wardii (Hook.) grown in $\mathrm{Pb}$-contaminated soils. Journal of Environmental Management. 2016, volumen 182, (94-100).

10. Rizzi L., Petruzzelli G., Poggio G., Guidi G. V. Soil physical changes and plant availability of Zn and $\mathrm{Pb}$ in a treatability test of phytostabilization. Chemosphere. 2004, volumen 57, (1039-1046). 


\section{CONGRESO IBÉRICO DE AGROINGENIERÍA \\ $X$ CONGRESSO IBÉRICO DE AGROENGENHARIA \\ 3-6 septiembre 2019, Huesca - España}

11. Bernal M., Clemente R., Walker D. J. The role of organic amendments in the bioremediation of heavy metal-polluted soils. Environmental Research at the Leading Edge. 2007, (1-57).

12. Renella G. et al. Long-term effects of aided phytostabilisation of trace elements on microbial biomass and activity, enzyme activities, and composition of microbial community in the Jales contaminated mine spoils. Environmental Pollution. 2008, volumen 152, (702-712).

13. Kumpiene J., Lagerkvist A., Maurice C. Stabilization of $\mathrm{As}, \mathrm{Cr}, \mathrm{Cu}, \mathrm{Pb}$ and $\mathrm{Zn}$ in soil using amendments - A review. Waste Management. 2008, volumen 28, (215-225).

14. Alvarenga P. et al. Assessment of chemical, biochemical and ecotoxicological aspects in a mine soil amended with sludge of either urban or industrial origin. Chemosphere. 2008, volumen 72, (17741781).

15. Alvarenga P. et al. Organic residues as immobilizing agents in aided phytostabilization: (II) Effects on soil biochemical and ecotoxicological characteristics. Chemosphere. 2009, volumen 74, (13011308).

16. Laperche V., Logan T. J., Gaddam P., Traina S. J. Effect of apatite amendments on plant uptake of lead from contaminated sail. Environmental Science and Technology. 1997, volumen 31, (2745-2753).

17. Cobb G. P., Sands K., Waters M., Wixson B. G., Dorward-King E. Accumulation of heavy metals by vegetables grown in mine wastes, 2000.

18. Conesa H. M., Pérez-Chacón J. A., Arnaldos R., Moreno-Caselles J., Faz-Cano Á. In situ heavy metal accumulation in lettuce growing near a former mining waste disposal area: Implications for agricultural management. Water, Air, and Soil Pollution. 2010, volumen 208, (377-383).

19. Peech M. Hydrogen Ion Activity. Black CA (ed) Methods of soil analysis. 1965, volumen Part 2, (914926).

20. United States Environmental Protection Agency (US-EPA) SW-846 Test Method 3051A: Microwave Assisted Acid Digestion of Sediments, Sludges, Soils, and Oils. US-EPA online. 2007, volumen вы12y, (245).

21. Tessier A., Campbell P. G. C., Bisson M. Sequential extraction procedure for the speciation of particulate trace metals. Analytical Chemistry. 1979, volumen 51, (844-851).

22. Li X., Coles B. J., Ramsey M. H., Thornton I. Sequential extraction of soils for multielement analysis by ICP-AES. Chemical Geology. 1995, volumen 124, (109-123).

23. Shiowatana J., McLaren R. G., Chanmekha N., Samphao A. Fractionation of Arsenic in Soil by a Continuous-Flow Sequential Extraction Method. Journal of Environment Quality. 2010, volumen 30, (1940).

24. Ghosh M., Singh S. P. A comparative study of cadmium phytoextraction by accumulator and weed species. Environmental Pollution. 2005, volumen 133, (365-371).

25. Barman S. C., Sahu R. K., Bhargava S. K., Chaterjee C. Distribution of Heavy Metals in Wheat, Mustard, and Weed Grown in Field Irrigated with Industrial Effluents. Bulletin of Environmental Contamination and Toxicology. 2000, volumen 64, (489-496).

26. Gupta S., Nayek S., Saha R. N., Satpati S. Assessment of heavy metal accumulation in macrophyte, agricultural soil, and crop plants adjacent to discharge zone of sponge iron factory. Environmental Geology. 2008, volumen 55, (731-739).

27. Zhao F., Hamon R. E., Lombi E., McLaughlin M. J., McGrath S. P. Characteristics of cadmium uptake in two contrasting ecotypes of the hyperaccumulator Thlaspi caerulescens. Journal of Experimental Botany. 2002, volumen 53, (535-543).

28. Lasat M. M., Pence N. S., Garvin D. F., Ebbs S. D., Kochian L. V Molecular physiology of zinc transport in the Zn hyperaccumulator Thlaspi caerulescens. Journal of experimental botany. 2000, volumen 51, (71-9).

29. Audet P., Charest C. Heavy metal phytoremediation from a meta-analytical perspective. Environmental Pollution. 2007, volumen 147, (231-237).

30. Brooks R. R.Ed. Plants that hyperaccumulate heavy metals: their role in phytoremediation, microbiology, archaeology, mineral exploration and phytomining. Wallingford, UK: CAB INTERNATIONAL, 1998. 


\section{CONGRESO IBÉRICO DE AGROINGENIERÍA \\ X CONGRESSO IBÉRICO DE AGROENGENHARIA \\ 3 - 6 septiembre 2019, Huesca - España}

31. Parra A., Zornoza R., Conesa E., Gómez-López M. D., Faz A. Evaluation of the suitability of three Mediterranean shrub species for phytostabilization of pyritic mine soils. CATENA. 2016, volumen 136, (59-65).

32. Fuentes D., Disante K. B., Valdecantos A., Cortina J., Vallejo V. R. Sensitivity of Mediterranean woody seedlings to copper, nickel and zinc. Chemosphere. 2007, volumen 66, (412-420).

33. Wang G., Zhang S., Zhong Q., Peijnenburg W. J. G. M., Vijver M. G. Feasibility of Chinese cabbage (Brassica bara) and lettuce (Lactuca sativa) cultivation in heavily metals-contaminated soil after washing with biodegradable chelators. Journal of Cleaner Production. 2018, volumen 197, (479-490).

34. Kabata-Pendias A., Pendias H. Trace elements in Soils and Plants. CRC Press LLC, 2001. 